Original Research Paper

\title{
Optimization of Ultrasonic/Microwave Assisted Extraction (UMAE) and Rheological Properties of Polysaccharides from Auricularia polytricha
}

\author{
Ze Zhang, Xiao Ding, Ziyi Xu, Xinyi Wu, Chenzhi Yang, Jing Hao and Yiyong Chen \\ School of Biology and Food Engineering, Changshu Institute of Technology, Changshu 215500, China
}

\author{
Article history \\ Received: 17-12-2019 \\ Revised: 21-01-2020 \\ Accepted: $12-03-2020$ \\ Corresponding Author: \\ Yiyong Chen \\ School of Biology and Food \\ Engineering, Changshu \\ Institute of Technology, \\ Changshu 215500, China \\ E-mail: greenpop6688@126.com
}

\begin{abstract}
Polysaccharides from Auricularia polytricha (APPS) were extracted using Ultrasonic/Microwave Assisted Extraction (UMAE) technology. UMAE conditions of APPS were optimized with Response Surface Methodology (RSM). Rheological properties of APPS were investigated. The results suggested that the optimal UMAE conditions for APPS were ratio of water to raw material $22(\mathrm{~mL} / \mathrm{g})$, extraction time 15.3 min and microwave power 59W. APPS solution exhibited Newtonian flow behavior at low concentration, formed entanglement network at lower concentration and established weak gel structures with solid-like properties at higher concentration. The viscosity of APPS solutions decreased when temperature increased. The viscosity, storage $\left(\mathrm{G}^{\prime}\right)$ and loss $\left(\mathrm{G}^{\prime \prime}\right)$ modulus of APPS solution increased when sucrose concentration increased. Heating time and freeze-thaw changes had no significant effect on rheological properties of APPS. The results suggested that UMAE was a suitable and efficient method for APPS extraction and APPS had potential as a novel food additive hydrocolloid in food industry.
\end{abstract}

Keywords: Auricularia polytricha, Polysaccharide, Ultrasonic/Microwave Assisted Extraction, Rheological Properties

\section{Introduction}

Mushrooms have been considered as edible and medicinal resources for thousands of years and many biologically active molecules have been isolated and identified from mushrooms (Wang et al., 2018). In addition, many mushrooms have been shown to contain bioactive polysaccharides in fruiting bodies (Chen et al., 2008; Zhao et al., 2010).

Auricularia polytricha belonging to the Auriculariaceae family is widely distributed in many places in China, such as Hebei, Heilongjiang, Anhui, Jiangxi and Guangdong. It has high contents of carbohydrates in the fruiting bodies traditionally used as food and medicine in China (Fan et al., 2006) and has lowering blood-fat, antioxidant, antitumor, antinociceptive and immunomodulatory activities (Mau et al., 2001).

In recent years, various new extraction techniques for extracting polysaccharides including Hot Water Extraction (HWE), Ultrasonic Assisted Extraction (UAE) and Microwave Assisted Extraction (MAE) have been developed (You et al., 2014). HWE requires high temperature and long extraction time (Li et al., 2007).
UAE has the benefits of mass transfer enhancement, cell destruction, increased permeability and capillary effects (Hmelkov et al., 2018). The main advantage of MAE is saving time (Zhang and Liu, 2008). Therefore, to take full advantage of the cavitation of ultrasonic vibration and the high-energy effect of microwave, combining ultrasonic and microwave extraction technology to extract polysaccharides is the future development trend. However, to date, there have been no reports of Ultrasound/Microwave Assisted Extraction (UMAE) of polysaccharides from A. polytricha (APPS).

Polysaccharides can be used as emulsifiers and thickeners in the food industry to improve the appearance characteristics of food. As an important feature of polysaccharides, rheological behavior not only affects the manufacture, storage and texture characteristics of food greatly, but also has an intrinsic relationship with molecular structure and chain conformation (Huang et al., 2009; Xu et al., 2008).

Polysaccharides as a colloidal substance are involved in the rheological properties in many practical applications. Therefore, an important prerequisite for 
expanding the application range of polysaccharides as a food additive hydrocolloid is to reveal the rheological properties of polysaccharides. APPS had anticancer activities (Song and Du, 2010), antitumor effects (Song and $\mathrm{Du}, 2012$ ), antioxidant (Chen and Xue, 2018) and antihypercholesterolemic effect (Zhao et al., 2015). However, no rheological properties of APPS have been reported in the literature.

Based on the above analysis, the present study aimed to optimize the UMAE conditions for APPS using response Surface Analysis Methodology (RSM) for finding a suitable and efficient method for APPS extraction. In addition, rheological properties of APPS were also investigated to explore further the applications and the functional properties as a novel food additive hydrocolloid in food industry.

\section{Materials and Methods}

\section{Materials}

The dried fruiting bodies of $A$. polytricha were purchased from Changshu city, China. The dried materials were crushed into a fine powder in a high disintegrator (Micron Co. LTD, China) for the subsequent studies. Ethanol, phenol, sulphuric acid and glucose were purchased from the Chengdu Kelong Chemical Factory (Chengdu, China). All reagents were of analytical grade.

\section{Experimental}

\section{Extraction of APPS with UMAE}

Ultrasonic/microwave assisted extraction was performed using an UMAE apparatus (CW-2000, Shanghai Xintuo Microwave Instrument Co. Ltd. China) with maximal microwave power of $800 \mathrm{~W}$ at a frequency of $2450 \mathrm{MHz}$ and an ultrasonic transducer with a fixed power of $50 \mathrm{~W}$ at a frequency of $40 \mathrm{KHz}$ (fixed parameter of equipment). $2.0 \mathrm{~g}$ fine powder of $A$. polytricha and $100 \mathrm{~mL}$ deionized water were added into a $250 \mathrm{~mL}$ flask and then transferred into the chamber of the apparatus connected with condensing tubes.

Extraction of APPS with UMAE was performed under different extraction time and microwave power conditions. After the extraction was completed, the proteins in polysaccharide extract were separated for 6 times with sevage method. The polysaccharide extract was then centrifuged at $4500 \mathrm{rpm}$ for $10 \mathrm{~min}$. The supernatant was concentrated in a rotary evaporator at $50^{\circ} \mathrm{C}$ and then precipitated with four volumes of $95 \%$ ethanol for $48 \mathrm{~h}$ at $4{ }^{\circ} \mathrm{C}$. The precipitate was collected by centrifugation (5000 rpm, $15 \mathrm{~min}$ ), washed with pure ethanol and acetone and finally lyophilized to obtain APPS. With D-glucose as the standard, the total carbohydrate content of APPS was determined by phenol sulfuric acid colorimetric method (Dubois et al., 1956). Yield and purity of APPS were measured using the following equation:

$$
\begin{aligned}
& \text { APPS yield }=\frac{\text { weight of crude polysaccha ride extract }(g)}{\text { weight of each powder sample }(g)} \times 100 \% \\
& \text { APPS purity }=\frac{\text { weight of determined polysaccharide }(g)}{\text { weight of crude polysaccha ride extract }(g)} \times 100 \%
\end{aligned}
$$

\section{Experimental Design of RSM and Statistical Analysis}

Response Surface Methodology (RSM) is used to explore the effect of independent variables on the response within the investigation scope. Statistical techniques can be collected by RSM to design experiments, build models, evaluate the effects of factors and find the optimal conditions for factors to obtain the desirable responses (Li et al., 2002).

Independent variables and their ranges were selected based on our preliminary single tests. A central composite design with three independent variables $\left(\mathrm{X}_{1}\right.$, ratio of water to raw materials; $\mathrm{X}_{2}$, extraction time; $\mathrm{X}_{3}$, microwave power) (Table 1) was performed to optimize extraction conditions and investigate the influence of the above independent variables on the dependent variables (yield and purity of polysaccharides).

To predict the optimal parameters of UMAE of APPS, a second-order polynomial model was fitted to establish the relationship between the independent and response variables as shown below:

$$
Y=\beta_{0}+\sum_{i=1}^{3} \beta_{i} X_{i}+\sum_{i}^{3} \beta_{i i} X_{i}^{2}+\sum_{i=1}^{2} \sum_{j=i+1}^{3} \beta_{i j} X_{i} X_{j}
$$

where, $y_{k}$ is the response, $b_{k 0}, b_{k i}, b_{k i i}$ and $b_{k i j}$ are coefficients for intercept, linear, quadratic and interactive terms, respectively; $x_{i}, x_{i}^{2}$ and $x_{i} x_{j}$ represent linear, quadratic and interactive terms of coded independent variables, respectively.

Table 1: Independent variables and their levels in the response surface design

\begin{tabular}{llll}
\hline & & Coded factor level & \\
Independent variables & Symbol & -1 & 0 \\
\hline Ratio of water to raw material $(\mathrm{mL} / \mathrm{g})$ & $\mathrm{X}_{1}$ & 10 & 20 \\
Extraction time (min) & $\mathrm{X}_{2}$ & 10 & 15 \\
Microwave power $(\mathrm{W})$ & $\mathrm{X}_{3}$ & 20 & 50 \\
\hline
\end{tabular}


The experimental data was analyzed using the Design Expert program (version 8.0.6). The statistical significance of the coefficients in the regression equation was checked by the ANOVA program. When $\mathrm{p}<0.05$, the significance of all terms in the polynomial was considered statistically different.

\section{Chemical Composition Analysis of APPS}

The total carbohydrates were determined by the phenol-sulfuric acid colorimetric method with D-glucose as a standard at $490 \mathrm{~nm}$ (Dubois et al., 1956).

Determination of uronic acid content was performed by measuring the absorbance at $525 \mathrm{~nm}$ using a metahydroxybiphenyl colorimetric method with glucuronic acid as a standard (Wang et al., 2017). The protein content was measured based on the reported method (Vakondios et al., 2014).

\section{FT-IR Spectroscopy}

$1 \mathrm{mg}$ APPS was ground with dried potassium bromide $(\mathrm{KBr})$ powder and then pressed into pellets for the analysis. The IR spectrum was recorded in the wavenumber range of $400-4000 \mathrm{~cm}^{-1}$ on a Nicolet IS10 FT-IR spectrometer (Thermo Nicolet Co., USA).

\section{Rheological Properties of APPS}

The dried APPS powder was dissolved in distilled water and stirred at $25^{\circ} \mathrm{C}$ for $2 \mathrm{~h}$. Thus, aqueous APPS solution with various concentrations of 0.1 to $3.0 \%$ (W/V) was obtained for the steady shear tests. A rheometer (Physica MCR 301, Anton Paar, Austria) was used to perform stable shear and oscillation tests using a parallel plate (40 $\mathrm{mm}$ diameter, $1.0 \mathrm{~mm}$ gap). The stable shear viscosity of various APPS concentrations was measured at $25^{\circ} \mathrm{C}$. Viscoelasticity, storage modulus $\left(\mathrm{G}^{\prime}\right)$ and loss modulus (G") were measured using a small amplitude oscillation test at a frequency of 0.1 to $10 \mathrm{~Hz}$. A temperature ramp from 20 to $80^{\circ} \mathrm{C}$ was applied using a parallel plate at a rate of $10^{\circ} \mathrm{C} / \mathrm{min}$. All rheological measurements were performed at least three replicates and the obtained data was the average values.

The APPS solution $(0.5 \%, \mathrm{~W} / \mathrm{V})(10 \mathrm{~mL})$ was heated at $50^{\circ} \mathrm{C}$ for $20,40,60$ and 80 min in a water bath, respectively. In addition, the APPS solution $(0.5 \%, \mathrm{~W} / \mathrm{V})$ $(10 \mathrm{~mL})$ was refrigerated at $4^{\circ} \mathrm{C}$, frozen at eighteen degrees below zero $\left(-18^{\circ} \mathrm{C}\right)$ and left at room temperature for $40 \mathrm{~min}$, respectively. After the above two treatment, the shear rate dependency of APPS solution viscosity was measured at $25^{\circ} \mathrm{C}$ to investigate the effect of heating time and temperature on the rheological properties of APPS. After stirring at room temperature for $40 \mathrm{~min}, 5 \mathrm{~mL}$ sucrose solution with a concentration of $20 \%$ and $50 \%(\mathrm{~W} / \mathrm{V})$ was added into the APPS solution $(0.5 \%, \mathrm{~W} / \mathrm{V})(10 \mathrm{~mL})$, respectively. Then, the shear rate dependency of APPS solution viscosity was measured at $25^{\circ} \mathrm{C}$ to investigate effect of sucrose on rheological properties.

\section{Results and Discussion}

\section{Statistical Analysis and the Model Building}

Results of response surface analysis of the variation of the yield and the purity of APPS with ratio of water to raw material $\left(X_{1}\right)$, time $\left(X_{2}\right)$ and microwave power $\left(X_{3}\right)$ were presented in Table 2. The regression coefficients and model analysis of the two response variables were summarized in Table 3. A second-order polynomial model that establishes the relationship between independence and yield $\left(Y_{1}\right)$ and purity $\left(Y_{2}\right)$ was obtained as follows:

$$
\begin{aligned}
& Y_{1}=4.2+0.14 X_{1}+0.025 X_{2}-0.15 X_{3}-0.36 X_{1}^{2} \\
& +0.073 X_{1} X_{2}-0.027 X_{1} X_{3}-0.39 X_{2}^{2}-0.089 X_{2} X_{3}-0.61 X_{3}^{2} \\
& Y_{2}=49.01+1.06 X_{1}+0.33 X_{2}-0.16 X_{3}-1.49 X_{1}^{2}+0.37 X_{1} X_{2} \\
& -0.49 X_{1} X_{3}-1.81 X_{2}^{2}+0.020 X_{2} X_{3}-1.94 X_{3}^{2}
\end{aligned}
$$

As shown in Table 3, the proposed regression model for yield and purity was sufficient to have satisfactory $\mathrm{R}^{2}$ values (0.9692 and 0.9411 , respectively), which indicated that the experimental results were very consistent with the theoretical values predicted by the polynomial models. Linear $\left(X_{1}\right.$ and $\left.X_{3}\right)$ and quadratic coefficients $\left(X_{1}^{2}, X_{2}^{2}\right.$ and $\left.X_{3}^{2}\right)$ were significant for yield $(\mathrm{P}<0.05$ or $\mathrm{P}<0.01)$. From the model of purity, Linear $\left(X_{1}\right)$ and quadratic coefficients $\left(X_{1}^{2}, X_{2}^{2}\right.$ and $\left.X_{3}^{2}\right)$ were significant $(\mathrm{p}<0.01)$. The independent variables including ratio of water to material and microwave power were the most important factors with a greater effect on the yield of APPS. At the same time, ratio of water to material affected purity of APPS most obviously.

According to Equation (4) and (5), 3D response surface plots and 2D contour plots (Fig. 1) were obtained to evaluate effect of independent variables and their interaction on the yield and purity of APPS. It could be seen from Fig. 1A and 1B that when extraction time was constant $(15.3 \mathrm{~min})$, the yield of APPS gradually increased with the increase of ratio of water to raw material $\left(X_{1}\right)$ and microwave power $\left(X_{3}\right)$ in the range of $10-22 \mathrm{~mL} / \mathrm{g}$ and $20-59.4 \mathrm{~W}$, respectively. However, when ratio of water to raw material and microwave power exceeded $22 \mathrm{~mL} / \mathrm{g}$ and $59.4 \mathrm{~W}$, respectively, the yield of APPS decreased slightly, which indicated that effect of ratio of water to raw material $\left(X_{1}\right)$ and microwave power $\left(X_{3}\right)$ on yield was significant. Similarly, effect of ratio of water to raw material $\left(\mathrm{X}_{1}\right)$ and microwave power $\left(\mathrm{X}_{3}\right)$ on purity was also significant (Fig. 1C and 1D). 
Ze Zhang et al. / American Journal of Biochemistry and Biotechnology 2020, 16 (1): 112.124 DOI: 10.3844/ajbbsp.2020.112.124

Table 2: Results of response surface analysis of the variation of the yield and the purity of polysaccharides from Auricularia polytricha with ratio of water to raw material $\left(\mathrm{X}_{1}\right)$, time $\left(\mathrm{X}_{2}\right)$ and microwave power $\left(\mathrm{X}_{3}\right)$

\begin{tabular}{lrrrrr}
\hline Number & $\mathrm{X}_{1}$ & $\mathrm{X}_{2}$ & $\mathrm{X}_{3}$ & Yield (\%) & Purity $(\%)$ \\
\hline 1 & -1 & -1 & 0 & 3.21 & 44.37 \\
2 & -1 & 1 & 0 & 3.49 & 44.34 \\
3 & 1 & 1 & 0 & 3.60 & 46.34 \\
4 & 1 & 1 & 0 & 3.59 & 47.78 \\
5 & 0 & -1 & -1 & 3.22 & 44.67 \\
6 & 0 & -1 & 1 & 3.28 & 45.22 \\
7 & 0 & 1 & -1 & 3.36 & 45.26 \\
8 & 0 & 1 & 1 & 3.06 & 45.89 \\
9 & -1 & 0 & -1 & 3.30 & 44.94 \\
10 & 1 & 0 & -1 & 3.69 & 47.45 \\
11 & -1 & 0 & 1 & 2.87 & 44.69 \\
12 & 1 & 0 & 1 & 3.14 & 45.23 \\
13 & 0 & 0 & 0 & 4.16 & 48.98 \\
14 & 0 & 0 & 0 & 4.19 & 49.68 \\
15 & 0 & 0 & 0 & 4.20 & 48.48 \\
\hline
\end{tabular}

Table 3: Regression coefficient and analysis of the model for two response variables

\begin{tabular}{lcc}
\hline coefficient & Yield $(\%)$ & Purity $(\%)$ \\
\hline $\mathrm{b}_{0}$ & 4.22 & 49.01 \\
$\mathrm{~b}_{1}$ & $0.14^{\mathrm{a}}$ & $1.06^{\mathrm{b}}$ \\
$\mathrm{b}_{2}$ & 0.025 & 0.33 \\
$\mathrm{~b}_{3}$ & $-0.15^{\mathrm{a}}$ & -0.16 \\
$\mathrm{~b}_{11}$ & $-0.36^{\mathrm{a}}$ & $-1.49^{\mathrm{b}}$ \\
$\mathrm{b}_{12}$ & 0.073 & 0.37 \\
$\mathrm{~b}_{13}$ & -0.027 & -0.49 \\
$\mathrm{~b}_{22}$ & $-0.39^{\mathrm{a}}$ & $-1.81^{\mathrm{b}}$ \\
$\mathrm{b}_{23}$ & -0.089 & 0.020 \\
$\mathrm{~b}_{33}$ & $-0.61^{\mathrm{b}}$ & $-1.94^{\mathrm{b}}$ \\
$\mathrm{R}^{2}$ & 0.9692 & 0.9411 \\
\hline
\end{tabular}

Note: 'a' means $\mathrm{P}<0.05$; 'b' means $\mathrm{P}<0.01$.

A

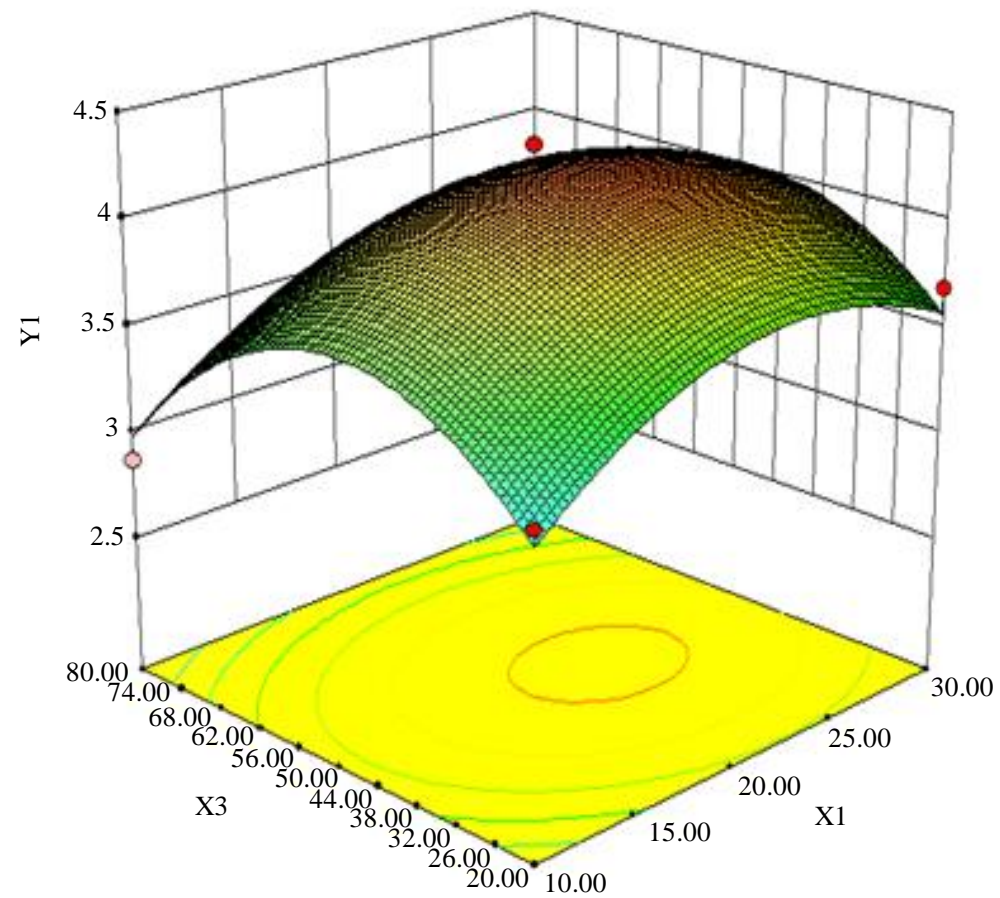


Ze Zhang et al. / American Journal of Biochemistry and Biotechnology 2020, 16 (1): 112.124 DOI: 10.3844/ajbbsp.2020.112.124

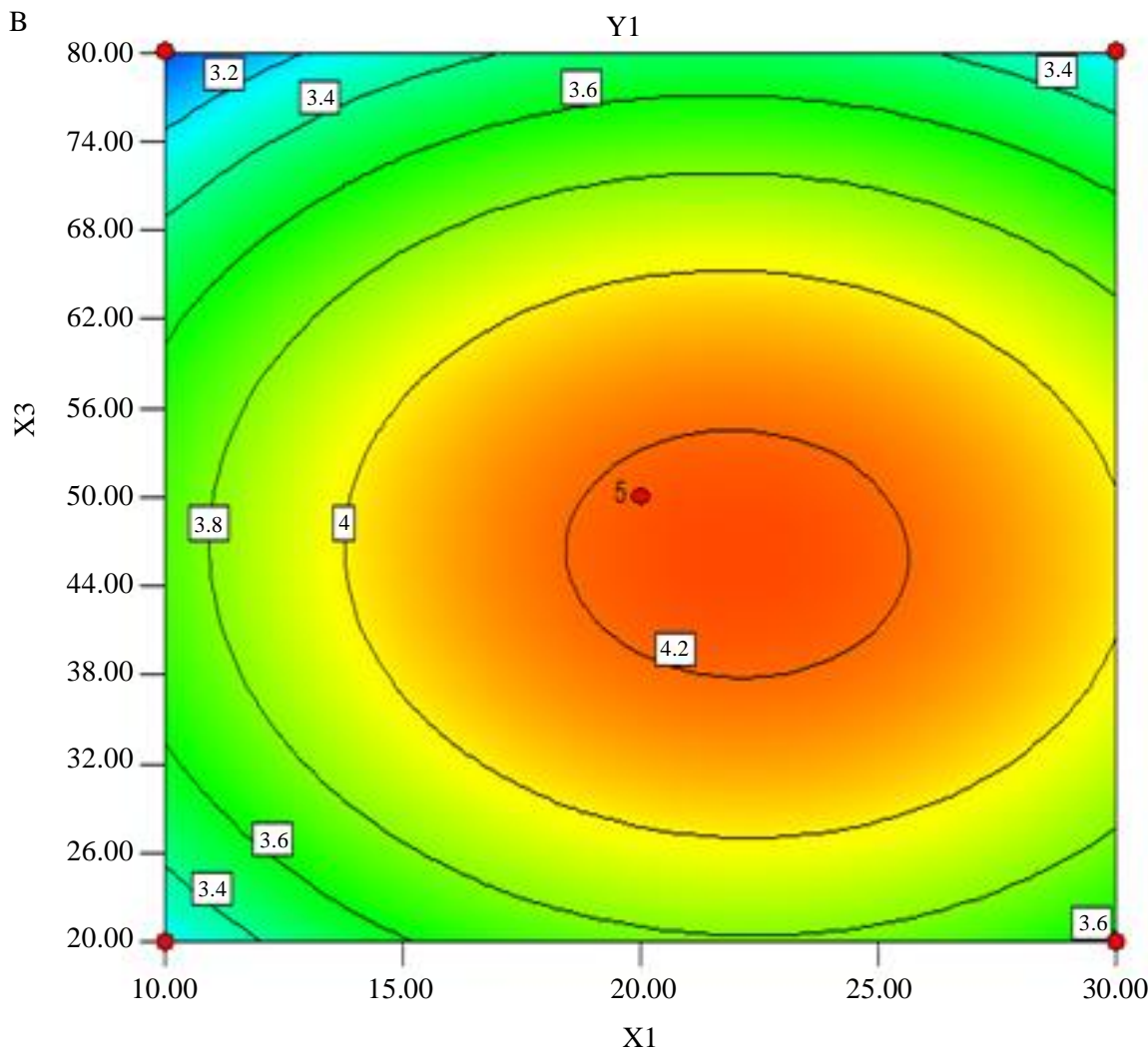

C

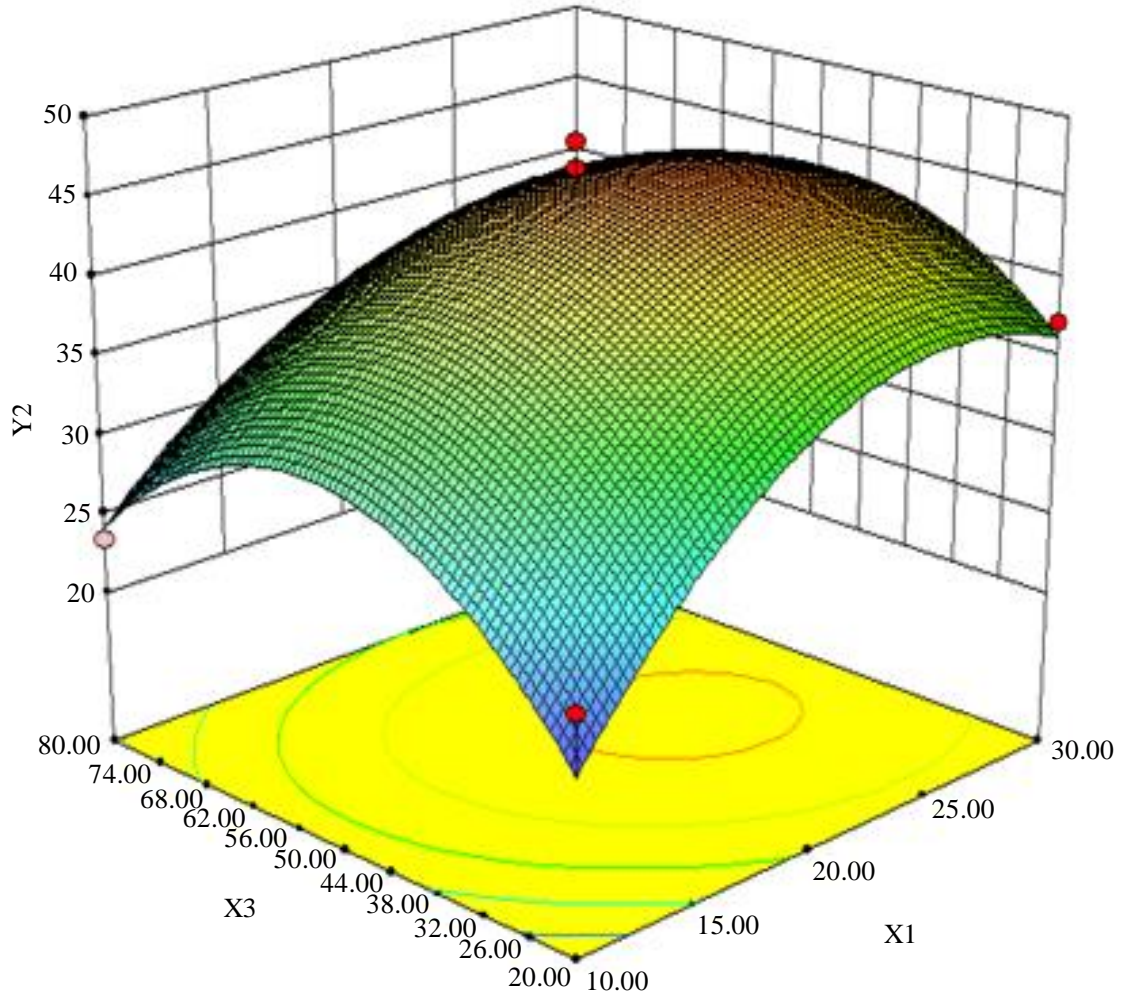


$\mathrm{D}$

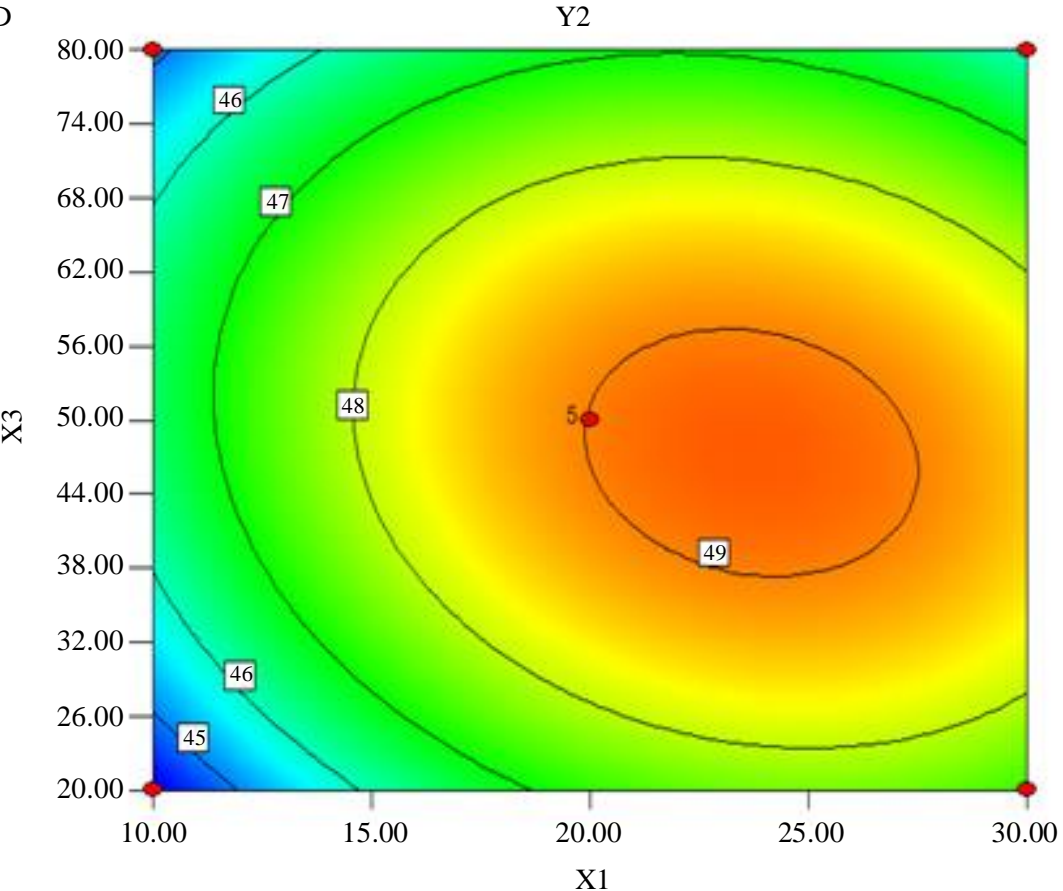

Fig. 1: Response surface (A and $C$ ) and contour (B and D) plots showing the effect of solid/water ratio and microwave power (extraction time $15.3 \mathrm{~min})$ on yield $\left(\mathrm{Y}_{1}\right)$ and purity $\left(\mathrm{Y}_{2}\right)$ of APPS

Optimization of UMAE Process of APPS and Validation of the Models

Based on the response surface plots, contour plots and analysis of variance, effect of extraction conditions on yield and purity of APPS was optimized and analyzed. The optimal UMAE process of APPS was obtained as follows: Ratio of water to raw material was $22 \mathrm{~mL} / \mathrm{g}$, extraction time was $15.3 \mathrm{~min}$ and microwave power was $59 \mathrm{~W}$.

To verify the adequacy of the model equations, three verification experiments were performed under optimal conditions as described above. The average yield and purity of APPS were $4.10 \%$ and $47.98 \%$, which were in good agreement with the predicted values $(4.12 \%$ and $48.92 \%)$. The results showed that the regression models were accurate and sufficient for UMAE of APPS.

\section{Chemical Analysis of APPS}

In the present study, APPS were extracted by UMAE under the optimal extraction conditions. Chemical analysis of APPS was performed. The result showed that content of total carbohydrate and uronic acid in APPS was $78.3 \pm 1.57 \%(\mathrm{~W} / \mathrm{W})$ and $19.5 \pm 1.21 \%(\mathrm{~W} / \mathrm{W})$, respectively. However, no proteins were found.

\section{FT-IR Spectroscopy}

FT-IR analysis of APPS was carried out and the result was shown in Fig. 2. A broad and strong absorption peak around $3434 \mathrm{~cm}^{-1}$ was due to the presence of the hydroxyl groups stretching vibration. A weak-CH stretching peak near $2949 \mathrm{~cm}^{-1}$ existed. A peak at $1740 \mathrm{~cm}^{-1}$ suggested carboxyl group existed. A absorption peak at $1633 \mathrm{~cm}^{-1}$ attributed to carboxyl group in uronic acid. In addition, the absorptions at 1026 and $1106 \mathrm{~cm}^{-1}$ indicated the presence of $\alpha$-pyranose ring (Wang et al., 2014).

\section{Rheological Properties of Apps}

\section{Flow Behavior of APPS}

Steady shear flow curve of APPS solution with different concentration $(0.1,0.2,0.5,1.0,2.0$ and $3.0 \%$, $\mathrm{W} / \mathrm{V})$ at $25^{\circ} \mathrm{C}$ was illustrated in Fig. 3. When the shear rate increased, the apparent viscosity of APPS solution with high concentration $(1.0,2.0$ and $3.0 \%, \mathrm{~W} / \mathrm{V}$, respectively) decreased significantly, which suggested that APPS solution exhibited pseudoplastic flow behavior at high shear rate region (shear rate $>25 \mathrm{~s}^{-1}$ ). However, at low concentration $(0.1,0.2$ and $0.5 \%$, respectively), APPS solution with relatively low apparent viscosity had almost Newtonian flow behavior.

At a specified shear rate, apparent viscosity increased when APPS concentration increased. Interpenetration of polymer chains can form a dynamic 'entangled' network structure in concentrated solutions. New interactions between different chains replaced those entanglements destroyed by the imposed deformation at low rates of shear and there is no net change in the degree of 
entanglement, so the viscosity is not lowered (Wu et al., 2012). Shear thinning occurs when the externally applied damage rate becomes larger than the formation rate of new entanglements. In this case, the density of the temporarily formed "crosslinking" of the network is depleted and thus the viscosity is lowered (Morris et al., 1981). Therefore, the crosslink density decreased further with the increase of shear rate, which could be proved by a decrease in apparent viscosity.

\section{Viscoelastic Behavior of APPS}

Because polysaccharides are viscoelastic materials with solid and liquid properties, the moduli $G^{\prime}$ and $G^{\prime \prime}$ refer to the elastic and viscous properties of a given material, respectively. The advantages of solid or liquid properties of a sample can be quantified by dynamic measurements. The frequency dependence of $G^{\prime}$ and $G^{\prime \prime}$ can be characterized by four most common classifications of dispersions, such as a dilute solution, an entangled network, a weak gel and a strong gel (Sun et al., 2014). A dilute solution shows $\mathrm{G}^{\prime \prime}$ higher than $\mathrm{G}^{\prime}$ over the entire frequency range (Nishinari, 1997). An entangled network shows that the $G^{\prime}$ and $G^{\prime \prime}$ curves intersect in the test frequency range, indicating that there is a clear trend toward more solid-like behavior at higher frequencies (Xu et al., 2008). The weak gel indicates that $\mathrm{G}^{\prime}$ is larger than $\mathrm{G}^{\prime \prime}$ and that they are almost parallel to each other. Strong gel also shows that $\mathrm{G}^{\prime}$ is greater than $\mathrm{G}^{\prime \prime}$, but the slope of the $\mathrm{G}^{\prime}$ curve is close to 0 and $\mathrm{G}^{\prime \prime}$ has a minimum at the intermediate frequencies (Moreira et al., 2014).

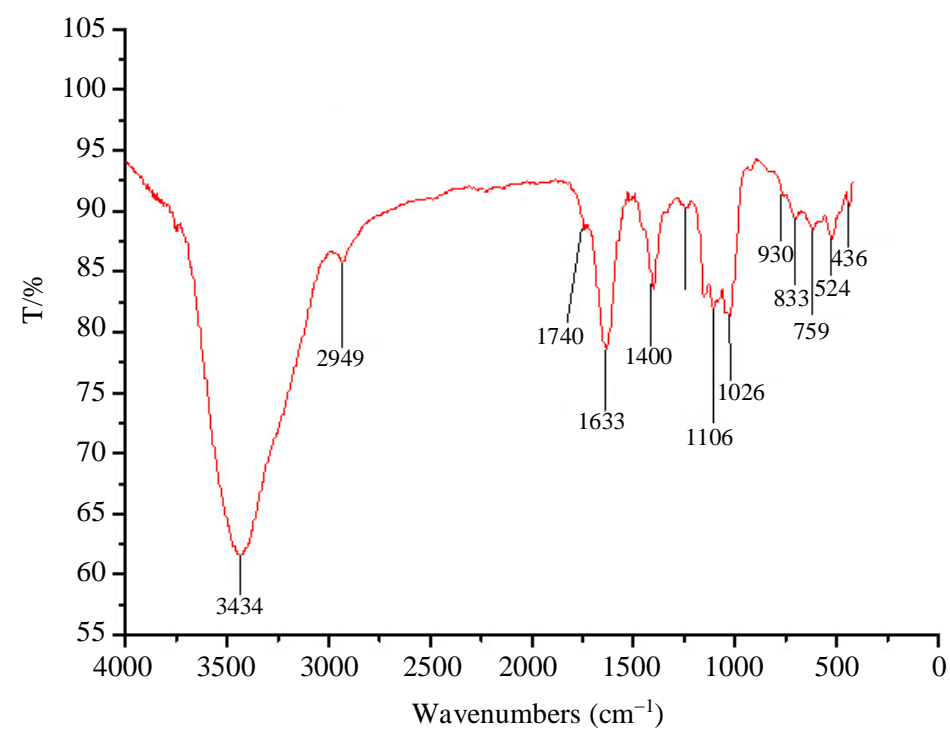

Fig. 2: FT-IR spectrum of APPS

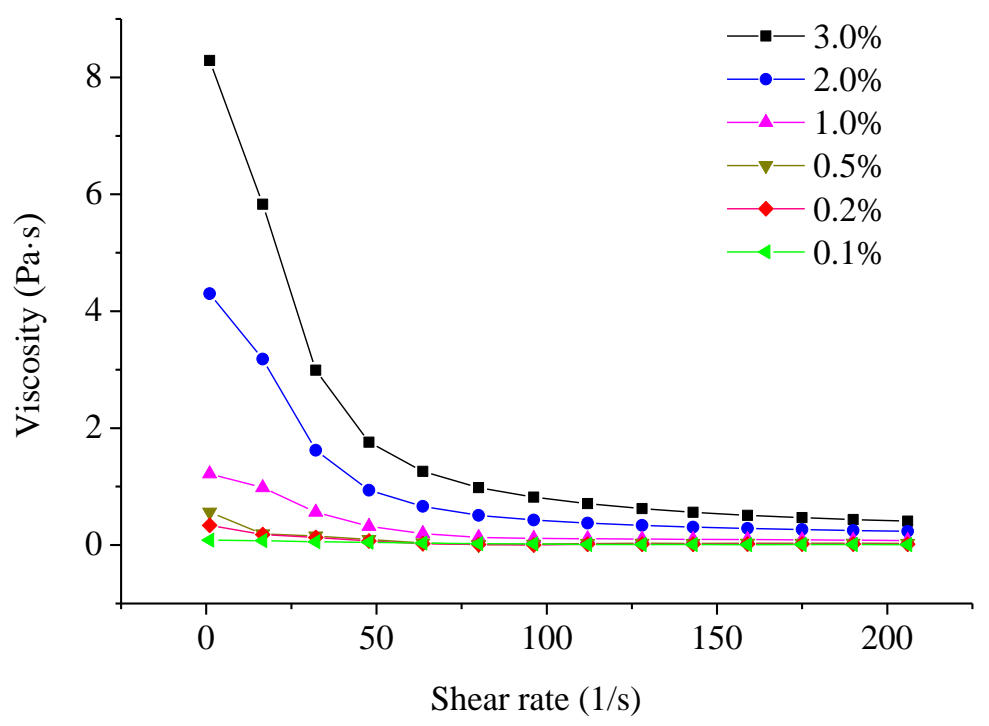

Fig. 3: Steady shear flow curves of different concentration of APPS at $25^{\circ} \mathrm{C}$ 
Based on analysis of frequency dependence of storage $\left(\mathrm{G}^{\prime}\right)$ and loss $\left(\mathrm{G}^{\prime \prime}\right)$ modulus of APPS solutions at $25^{\circ} \mathrm{C}$, the viscoelastic behavior of APPS solutions $(0.5-$ $3.0 \%, \mathrm{~W} / \mathrm{V}$ ) was shown in Fig. 4. It was observed that in the low frequency range $(\mathrm{f}<3 \mathrm{~Hz})$, the $\mathrm{G}^{\prime}$ and $\mathrm{G}^{\prime \prime}$ of all APPS solutions increased with the increase of frequency. The concentration of APPS (c) had a significant effect on $\mathrm{G}^{\prime}$ and $\mathrm{G}^{\prime \prime}$. When c was $0.5 \%$ or $1.0 \%$ and the frequency was lower than $6 \mathrm{~Hz}$, a crossover between $\mathrm{G}^{\prime}$ and $\mathrm{G}^{\prime \prime}$ was observed. When c increased, the cross point moved toward low frequency. $\mathrm{G}^{\prime}$ was always larger than $\mathrm{G}^{\prime \prime}$ over the entire frequency range and when c exceeded $2.0 \%$, the two moduli were almost parallel to each other. Based on the classifications mentioned above, $0.5 \%$ and $1.0 \%$ APPS solution formed an entangled network, APPS solution with higher $\mathrm{c}(\geq 2.0 \%)$ established weak gel structures with solid-like properties.

More interestingly, $\mathrm{G}^{\prime}$ dropped while $\mathrm{G}^{\prime \prime}$ increased at $c$ $(0.5 \%$ and $1.0 \%)$ when frequency exceeded $3 \mathrm{~Hz}$. The two moduli crossed at a certain frequency and the crossover of $G^{\prime}$ and $G^{\prime \prime}$ exhibited a shift to high frequency with the increase of $c$. The reason may be that the junctions formed by weak interactions between molecules were destroyed under high frequency oscillatory stress, resulting in decrease in the elastic response $G^{\prime}$ and increase in the viscous response $\mathrm{G}^{\prime \prime}$. The number and strength of intermolecular junctions increases with the increase of $c$. Therefore, the frequency oscillation stress is high enough to destroy these junctions, which leads to an increase in the frequency of intersection of $\mathrm{G}^{\prime}$ and $\mathrm{G}^{\prime \prime}$ (Moreira et al., 2014). It had been reported that $G^{\prime}$ and $G^{\prime \prime}$ of the Ice Cream Mix (ICM) initially increased as the oscillation frequency increased and then decreased (Dogan et al.,
2013; Toker et al., 2013). Similar phenomenon was also found in polysaccharides from Ulva fasciata (Shao et al., 2015).

\section{Effect of Temperature on Rheological Property of APPS Snolutio}

Effect of temperature on rheological property of APPS solution $(0.5 \%$, w/v)was shown in Fig. 5A. The results suggested that the apparent viscosity of APPS decreased nonlinearly when solution temperature increased. An increase of solution temperature usually results in a rapid decrease in the ratio of the radius of gyration to the average molecular weight, which increases the flexibility and tightness of the molecular chain and reduces the apparent viscosity (Vardhanabhuti and Ikeda, 2006). The heating curve of $0.5 \%$ APPS solution was obtained at a heating rate of $10^{\circ} \mathrm{C} / \mathrm{min}$ at constant frequency $(1.59 \mathrm{~Hz})$ shown in Fig. $5 \mathrm{~B}$. Both the storage modulus $\left(\mathrm{G}^{\prime}\right)$ and the loss modulus $\left(G^{\prime \prime}\right)$ decreased slightly when temperature increased, but the amplitude of the change was small.

\section{Effect of Heating Time on APPS Viscosity}

Effect of heating time on APPS viscosity was shown in Fig. 6A. Under different heating time conditions, the viscosity of APPS decreased gradually with the increase of shear rate. The lowest and the highest viscosity was obtained when heating time was $40 \mathrm{~min}$ and $60 \mathrm{~min}$, respectively. However, the heating time had little effect on the viscosity of APPS, which was beneficial to the heat treatment for APPS as a novel food additive in food processing.

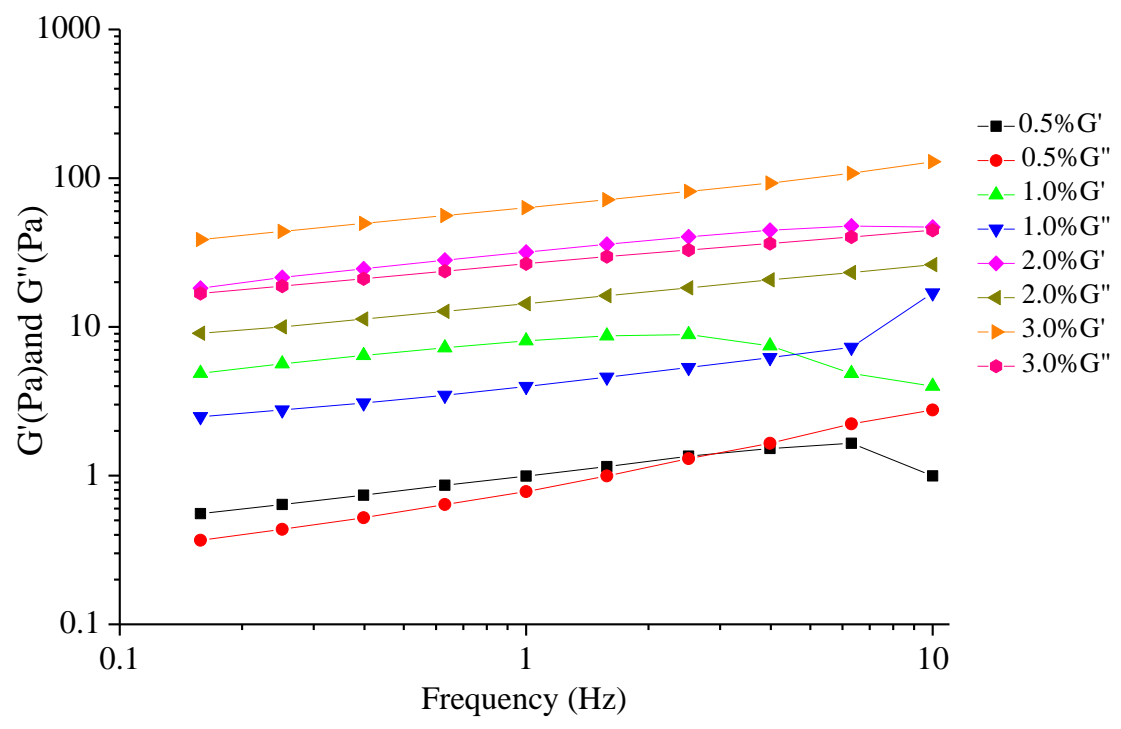

Fig. 4: Frequency dependence of storage $\left(\mathrm{G}^{\prime}\right)$ and loss $\left(\mathrm{G}^{\prime \prime}\right)$ modulus of APPS solutions at different concentrations at $25^{\circ} \mathrm{C}$ 

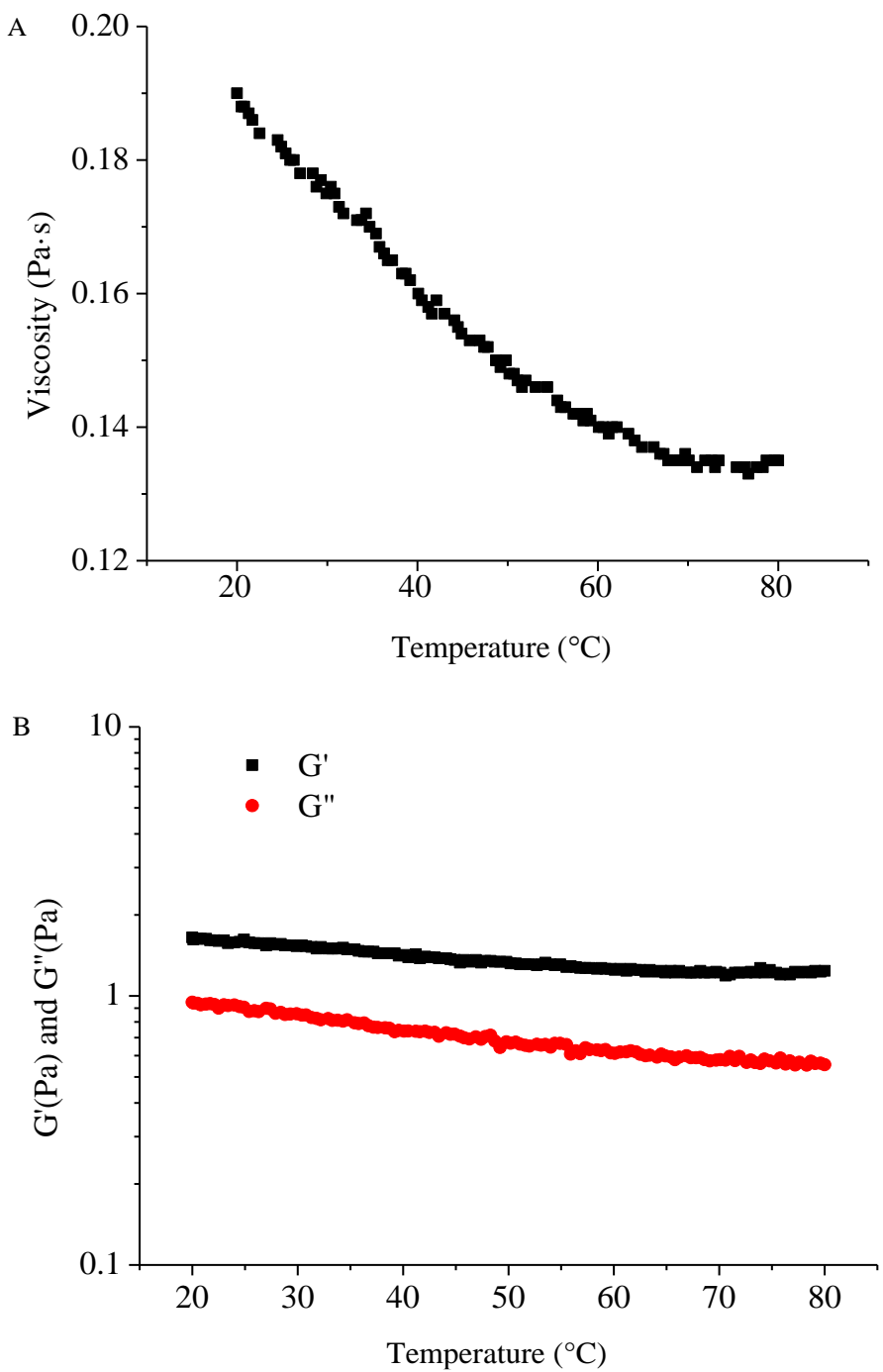

Fig. 5: Effect of temperature on viscosity of APPS solution (0.5\%) (A) and changes of storage $\left(\mathrm{G}^{\prime}\right)$ and loss $\left(\mathrm{G}^{\prime \prime}\right)$ modulus of APPS solution $(0.5 \%)$ at a heating rate $10^{\circ} \mathrm{C} / \mathrm{min}$ from 20 to $80^{\circ} \mathrm{C}(\mathrm{B})$

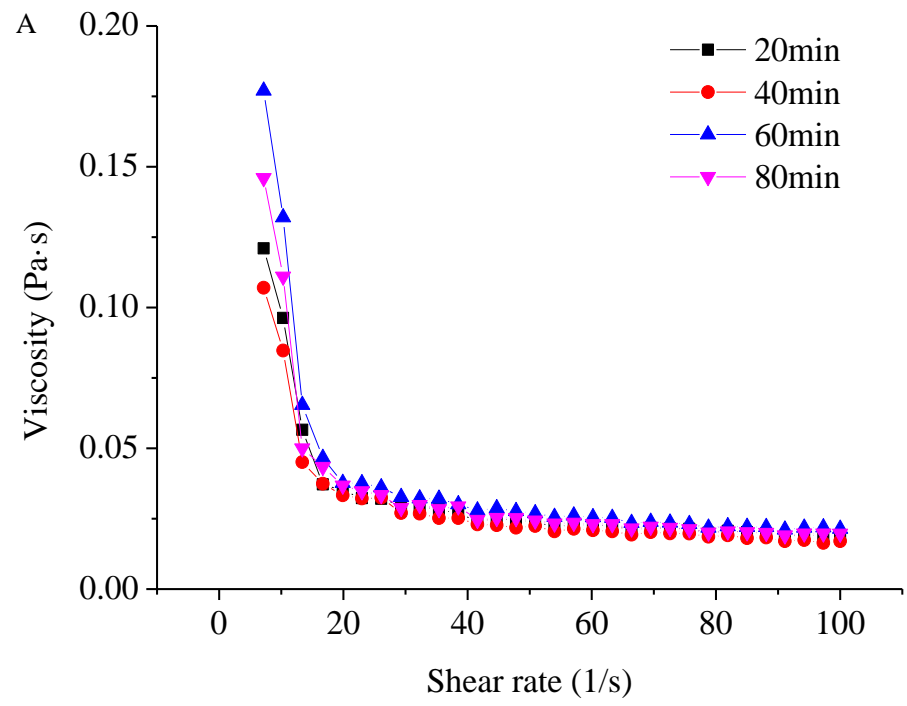



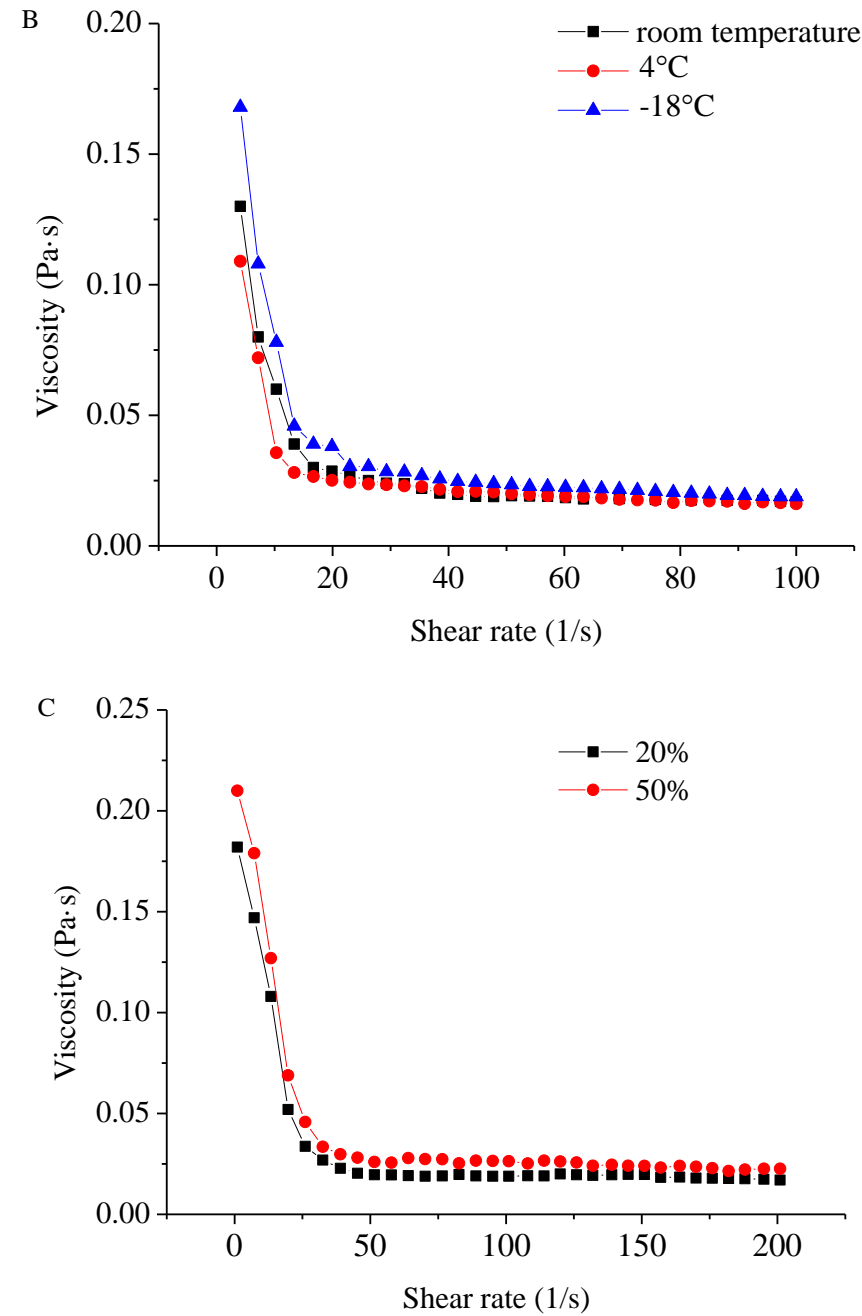

Fig. 6: Shear rate dependency of viscosity of APPS with different factors. (A) heating time; (B) freeze-thawing and (C) sucrose

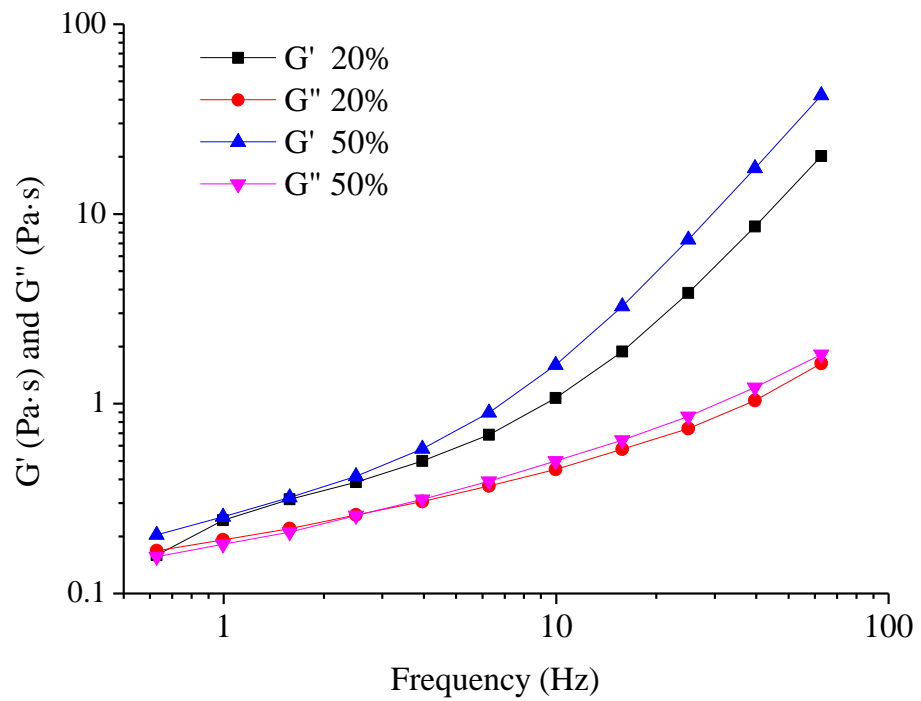

Fig. 7: Frequency dependence of storage $\left(\mathrm{G}^{\prime}\right)$ and loss $\left(\mathrm{G}^{\prime \prime}\right)$ modulus of APPS with $20 \%$ and $50 \%$ sucrose at $25^{\circ} \mathrm{C}$ 


\section{Effect of Freeze-Thawing on APPS Viscosity}

Effect of freeze-thawing on APPS viscosity was shown in Fig. 6B. Under different temperature conditions, the viscosity of APPS decreased gradually with the increase of shear rate. The viscosity of APPS decreased at $4^{\circ} \mathrm{C}$ refrigeration, while the viscosity increased under the frozen $\left(-18^{\circ} \mathrm{C}\right)$ compared with that at room temperature. However, the change of viscosity of APPS under different temperature was small, which indicated that the freeze-thawing change has little effect on the viscosity of APPS. Some hydrocolloids such as xanthan gum, guar gum exhibited excellent stability during freeze-thaw cycling known as freeze-thaw stability (Vardhanabhuti and Ikeda, 2006). Our results indicated that APPS could be used as a novel food additive in food products that required stability after freezing.

\section{Effect of the Sucrose on APPS Viscosity}

Effect of the sucrose on APPS viscosity was shown in Fig. 6C. Under different sucrose concentration (20\% and $50 \%$ ) conditions, the viscosity of APPS decreased gradually with the increase of shear rate. The viscosity of APPS increased with the increase of sucrose concentration. However, the change of viscosity of APPS under different sucrose concentration was small. The frequency dependence of storage $\left(\mathrm{G}^{\prime}\right)$ and loss $\left(\mathrm{G}^{\prime \prime}\right)$ modulus of APPS with $20 \%$ and $50 \%$ sucrose at $25^{\circ} \mathrm{C}$ was shown in Fig. 7. The storage $\left(\mathrm{G}^{\prime}\right)$ and loss $\left(\mathrm{G}^{\prime \prime}\right)$ modulus increased with the increase of sucrose concentration, which indicated that the elastic and gel structure of APPS were enhanced.

\section{Conclusion}

Ultrasonic/Microwave Assisted Extraction (UMAE) technology was applied to extract APPS. UMAE conditions of APPS were optimized with RSM on basis of single factor experiment as follows: Ratio of water to raw material $22 \mathrm{~mL} / \mathrm{g}$, extraction time $15.3 \mathrm{~min}$ and microwave power $59 \mathrm{~W}$. Chemical characterization analysis showed that APPS contained $78.3 \pm 1.57$ of total carbohydrate, $19.5 \pm 1.21 \%(\mathrm{w} / \mathrm{w})$ of uronic acid and no proteins. APPS had the general characteristic absorption peaks of polysaccharides by FT-IR analysis. APPS solution exhibited Newtonian flow behavior at low concentration (0.1, 0.2 and $0.5 \%$, respectively), formed entanglement network at lower concentration $(<2.0 \%)$ and established weak gel structures with solid-like properties at higher concentration $(\geq 2.0 \%)$. The viscosity of APPS solutions decreased with the increase of temperature. However, the viscosity, storage ( $\left.\mathrm{G}^{\prime}\right)$ and loss $\left(\mathrm{G}^{\prime \prime}\right)$ modulus of APPS solution increased with the increase of sucrose concentration, which indicated that the elastic and gel structure of APPS were enhanced. Heating time and freeze-thaw changes did not show a significant effect on rheological properties of APPS. The lowest and the highest viscosity was obtained when heating time was 40 min and $60 \mathrm{~min}$, respectively. Our results suggested that UMAE was a suitable and efficient method for APPS extraction and APPS had potential as a novel food additive hydrocolloid for application in food industry.

\section{Acknowledgement}

The authors are grateful for financial sponsored by Science and Technology Support Program (Agriculture) of Zhangjiagang City (ZKN1802).

\section{Author's Contributions}

Ze Zhang and Xiao Ding: Participated in all experiments and article writing; also responsible for data analysis.

Ziyi Xu and Xinyi Wu: Participated in optimization of UMAE conditions of APPS on basis of single factor experiment and rheological properties of APPS and data analysis.

Chenzhi Yang and Jing Hao: Participated in chemical characterization analysis and rheological properties of APPS and data processing.

Yiyong Chen: Participated in project design and experimental guidance of this manuscript.

\section{Ethics}

This article is original and contains unpublished material. The corresponding author confirms that all of the other authors have read and approved the manuscript and no ethical issues involved.

\section{References}

Chen, W., Z. Zhao, S.F. Chen and Y.Q. Li, 2008. Optimization for the production of exopolysaccharide from Fomes fomentarius in submerged culture and its antitumor effect in vitro. Bioresource Technol., 8: 3187-3194. DOI: 10.1016/j.biortech.2007.05.049

Chen, Y.Y. and Y.T. Xue, 2018. Purification, chemical characterization and antioxidant activities of a novel polysaccharide from Auricularia polytricha. Int. J. Biol. Macromol., 120: 1087-1092.

DOI: 10.1016/j.ijbiomac.2018.08.160

Dogan, M., A. Kayacier, O.S. Toker, M.T. Yilmaz and S. Karaman, 2013. Steady, dynamic, creep and recovery analysis of ice cream mixes added with different concentrations of xanthan gum. Food Bioprocess Tech., 6: 1420-1433. DOI: 10.1007/s11947-012-0872-z

Dubois, M., K.A. Gilles, J.K. Hamilton, P.A. Rebers and F. Smith, 1956. Colorimetric method for determination of sugars and related substances. Aanal. Chem., 28: 350-356. 
Fan, L., S. Zhang, L. Yu and L. Ma, 2006. Evaluation of antioxidant property and quality of breads containing Auricularia auricula polysaccharide flour. Food Chem., 3: 1158-1163.

DOI: 10.1016/j.foodchem.2006.03.017

Hmelkov, A.B., T.N. Zvyagintseva, N.M. Shevchenko, A.B. Rasin and S.P. Ermakova, 2018. Ultrasoundassisted extraction of polysaccharides from brown alga Fucus evanescens. Structure and biological activity of the new fucoidan fractions. J. Applied Phycol., 30: 2039-2046.

DOI: $10.1007 / \mathrm{s} 10811-017-1342-9$

Huang, Z.P., Y.N. Huang, X.B. Li and L.N. Zhang, 2009. Molecular mass and chain conformations of Rhizoma Panacis Japonici polysaccharides. Carbohyd. Polym., 3: 596-601. DOI: 10.1016/j.carbpol.2009.05.027

Li, C., J. Bai, Z. Cai and F. Ouyang, 2002. Optimization of a cultural medium for bacteriocin production by Lactococcus lactis using response surface methodology. J. Biotechnol., 93: 27-34.

DOI: $10.1016 / \mathrm{S} 0168-1656(01) 00377-7$

Li, J.W., S.D. Ding and X.L. Ding, 2007. Optimization of the ultrasonically assisted Extraction of polysaccharides from Zizyphus jujuba cv. jinsixiaozao. J. Food Eng., 80: 176-183.

DOI: 10.1016/j.jfoodeng.2006.05.006

Mau, J.L., G.R. Chao and K.T. Wu, 2001. Antioxidant properties of methanolic tracts from several ear mushrooms. J. Agr. Food Chem., 49: 5461-5467. DOI: $10.1021 /$ jf010637h

Moreira, H.R., F. Munarin, R. Gentilini, L. Visai and P.L. Granja et al., 2014. Injectable pectin hydrogels produced by internal gelation: $\mathrm{pH}$ dependence of gelling and rheological properties. Carbohyd. Polym., 103: 339-347.

DOI: $10.1016 /$ j.carbpol.2013.12.057

Morris, E.R., A.N. Cutler, S.B. Ross-Murphy, D.A. Rees and J. Price, 1981. Concentration and shear rate dependence of viscosity in random coil polysaccharide solutions. Carbohyd. Polym., 1: 5-21. DOI: 10.1016/0144-8617(81)90011-4

Nishinari, K., 1997. Rheological and DSC study of sol-gel transition in aqueous dispersions of industrially important polymers and colloids. Colloid. Poly. Sci., 12: 1093-107.

DOI: $10.1007 / \mathrm{s} 003960050189$

Song, G.L. and Q.Z. Du, 2010. Isolation of a polysaccharide with anticancer activity from Auricularia polytricha using high-speed countercurrent chromatography with an aqueous twophase system. J. Chromatogr. A, 1217: 5930-5934. DOI: 10.1016/j.chroma.2010.07.036
Song, G.L. and Q.Z. Du, 2012. Structure characterization and antitumor activity of an $\alpha \beta$-glucan polysaccharide from Auricularia polytricha. Food Res. Int., 45: 381-387.

DOI: $10.1016 /$ j.foodres.2011.10.035

Shao, P., J.M. Shao, L.F. Han, R.L. Lv and P.L. Sun, 2015. Separation, prelimnary characterrization and moisture-preserving activity of polysaccharides from Ulva fasciata. Int. J. Biol. Macromol., 72: 924-930.

DOI: 10.1016/j.ijbiomac.2014.09.048

Sun, F.Y., Q.L. Huang and J.Y. Wu, 2014. Rheological behaviors of an exopolysaccharide from fermentation medium of a Cordyceps sinensis fungus (Cs-HK1). Carbohyd. Polym., 114: 506-513. DOI: 10.1016/j.carbpol.2014.08.055

Toker, O.S., S.K. Araman, F. Yuksel, M. Dogan and A. Kayacier et al., 2013. Temperature dependency of steady, dynamic and creep-recovery rheological properties of ice cream mix. Food Bioprocess Tech., 11: 2974-2985. DOI: 10.1007/s11947-012-1005-4

Vakondios, N., E.E. Koukouraki and E. Diamadopoulos, 2014. Effluent Organic Matter (EfOM) characterization by simultaneous measurement of proteins and humic matter. Water Res., 63: 62-70. DOI: 10.1016/j.watres.2014.06.011

Vardhanabhuti, B. and S. Ikeda, 2006. Isolation and characterization of hydrocolloids from monoi (Cissampelos pareira) leaves. Food Hydrocolloid., 20: 885-891. DOI: 10.1016/j.foodhyd.2005.09.002

Wang, F.F., C. Shi, Y. Yang, Y. Fang and L. Sheng et al., 2018. Medicinal mushroom Phellinus igniarius induced cell apoptosis in gastric cancer SGC-7901 through a mitochondria-dependent pathway. Biomed. Pharmacother., 102: 18-25.

DOI: 10.1016/j.biopha.2018.03.038

Wang, Q.H., Y.P. Sun, B.Y. Yang, Z.B. Wang and Y.X. Liu et al., 2014. Optimization of polysaccharides extraction from seeds of pharbitis nil and its antioxidant activity. Carbohyd. Polym., 102: 460-466. DOI: 10.1016/j.carbpol.2013.11.068

Wang, X., L.H. Zhang, J.L. Wu, W.Q. Xu and X.Q. Wang et al., 2017. Improvement of simultaneous determination of neutral monosaccharides and uronic acids by gas chromatography. Food Chem., 220: 198-207.

DOI: 10.1016/j.foodchem.2016.10.008

Wu, Y., S.W. Cui, J.H. Wu, L.Z. Ai and Q. Wang et al., 2012. Structure characteristics and rheological properties of acidic polysaccharide from boat-fruited sterculia seeds. Carbohyd. Polym., 88: 926-930. DOI: 10.1016/j.carbpol.2012.01.035 
Xu, X.J., J. Xu, Y.Y. Zhang and L.N. Zhang, 2008. Rheology of triple helical lentinan in solution: Steady shear viscosity and dynamic oscillatory behavior. Food Hydrocolloid., 5: 735-741.

DOI: 10.1016/j.foodhyd.2007.02.010

You, Q.H., X.L. Yin, S.N. Zhang and Z.H. Jiang, 2014. Extraction, purification and antioxidant activities of polysaccharides from Tricholoma mongolicum Imai. Carbohyd. Polym., 99: 1-10.

DOI: 10.1016/j.carbpol.2013.07.088

Zhao, L., Y.H. Dong, G.T. Chen and Q.H. Hu, 2010. Extraction, purification, characterization and antitumor activity of polysaccharides from Ganoderma lucidum. Carbohyd. Polym., 3: 783-789. DOI: 10.1016/j.carbpol.2009.12.029
Zhang, L.F and Z.L. Liu, 2008. Optimization and comparison of Ultrasound/Microwave Assisted Extraction (UMAE) and Ultrasonic Assisted Extraction (UAE) of lycopene from tomatoes. Ultrason. Sonochem., 15: 731-737.

DOI: 10.1016/j.ultsonch.2007.12.001

Zhao, S., C.B. Rong, Y. Liu, F. Xu and C.L. Duan et al., 2015. Extraction of a soluble polysaccharide from Auricularia polytricha and evaluation of its anti-hypercholesterolemic effect in rats. Carbohyd. Polym., 122: 39-45.

DOI: $10.1016 /$ j.carbpol.2014.12.041 